\title{
Impact of a Clinical Trial Initiative on Clinical Trial Enrollment in a Multidisciplinary Prostate Cancer Clinic
}

\author{
Lydia T. Madsen, RN, MSNa; Deborah A. Kuban, MD; Seungtaek Choi, MD; John W. Davis, MD; \\ Jeri Kim, MDª Andrew K. Lee, MD, MPH ${ }^{\text {b }}$; Delora Domain, MS ${ }^{\mathrm{d}}$; Larry Levy, MS ${ }^{\mathrm{b}}$; Louis L. Pisters, MD; \\ Curtis A. Pettaway, MDc; John F. Ward, MDc Christopher Logothetis, MDª and \\ Karen E. Hoffman, MD, MPH ${ }^{\mathrm{b}}$
}

\begin{abstract}
Clinical oncology trials are hampered by low accrual rates, with fewer than $5 \%$ of adult patients with cancer treated on study. Clinical trial enrollment was evaluated at The University of Texas MD Anderson Cancer Center's Multidisciplinary Prostate Cancer Clinic (MPCC) to assess whether a clinical trial initiative, introduced in 2006, impacted enrollment. The trial initiative included posting trial-specific information in clinic, educating patients about appropriate clinical trial options during the treatment recommendation discussion, and providing patients with trial-specific educational information. The investigators evaluated the frequency of clinical trial enrollment for men with newly diagnosed prostate cancer seen in the MPCC from 2004 to 2008. Logistic regression evaluated the impact of patient characteristics and the clinical trial initiative on trial enrollment. The median age of the 1370 men was 64 years; $32 \%$ had low-risk, $49 \%$ had intermediate-risk, and $19 \%$ had high-risk disease. Overall, $74 \%$ enrolled in at least one trial and $29 \%$ enrolled in more than one trial. Trial enrollment increased from $39 \%$ before the initiative (127/326) to $84 \%(880 / 1044)$ after the trial initiative. Patient enrollment increased in laboratory studies (from $25 \%$ to $80 \%$ ), quality-of-life studies (from $10 \%$ to $26 \%$ ), and studies evaluating investigational treatments and systemic agents (from $6 \%$ to $15 \%$ ) after the trial initiative. In multivariate analysis, younger men $(P<.001)$ and men seen after implementation of the clinical trial initiative $(P<.001)$ were more likely to enroll in trials. Clinical trial enrollment in the MPCC was substantially higher than that seen nationally in adult patients with cancer, and enrollment rates increased after the introduction of a clinical trial initiative. ( J Natl Compr Canc Netw 2014;12:993-998)
\end{abstract}

\footnotetext{
From the Departments of a Genitourinary Medical Oncology, bRadiation Oncology, 'Urology, and Investigational Cancer Therapeutics, The University of Texas MD Anderson Cancer Center, Houston, Texas.

Submitted September 17, 2013; accepted for publication January 13, 2014

The authors have disclosed that they have no financial interests, arrangements, affiliations, or commercial interests with the manufacturers of any products discussed in this article or their competitors.

Correspondence: Lydia T. Madsen, MSN, RN, Department of Genitourinary Medical Oncology, The University of Texas MD Anderson Cancer Center, 1220 Holcombe Boulevard, Unit 1374, Houston, TX 77030. E-mail: Imadsen@mdanderson.org
}

An estimated 1 in 6 men in the United States will be diagnosed with prostate cancer in their lifetime. ${ }^{1}$ Although progress has been made, much still needs to be done to improve the diagnosis, treatment, survival, and quality of life for this patient population. Prostate cancer remains the second leading cause of cancer-related death among men in the United States, and prostate cancer survivors frequently experience long-term side effects of treatment. ${ }^{2,3}$ An obstacle to research progress is arguably the low rate of clinical trial enrollment. Recent reports estimate only $2 \%$ to $5 \%$ of adults diagnosed with cancer participate in clinical trials, although up to $20 \%$ of adults may be eligible for disease-specific clinical trial participation. ${ }^{4-6}$

One barrier to clinical trial enrollment is lack of awareness of available clinical trials. A survey of nearly 6000 patients with cancer found that $85 \%$ were either unaware or unsure that participation in clinical trials was an option, although $75 \%$ of these patients indicated a willingness to consider enrolling in a trial had they known it was an option. ${ }^{7}$ Lack of physician awareness of and time to discuss clinical trials also contribute to the limited patient awareness of available clinical trials and subsequent low clinical trial enrollment. ${ }^{5}$

A clinical trial initiative was introduced into MD Anderson Cancer Center's Multidisciplinary Prostate Cancer Clinic (MPCC) as a possible means to increase clinical trial enrollment and overcome these barriers. The initiative was based on the premise that providing an organized, methodical approach to incorporating clinical trial recommendations into an established clinic could facilitate greater patient understanding and consideration of clinical trial options. This article reports on the impact this initiative had on trial enrollment at a comprehensive cancer center. 


\section{Methods}

The MPCC opened in 2004 in the Genitourinary Center at The University of Texas MD Anderson Cancer Center. The clinic provides detailed education to newly diagnosed patients about their disease and appropriate treatment options. All patients meet with a urologist and radiation oncologist. A medical oncologist consultation is available for patients recommended for either systemic therapy or active surveillance. At the end of the clinic visit, patients receive both verbal and written detailed recommendations for treatment options.

A clinical trial initiative was introduced in the MPCC in 2006, which focused on informing patients of their clinical trial options and streamlining the referral process to educate patients on appropriate clinical protocols. Information about specific protocols and eligibility criteria were posted in the clinic area for physician reference. Trial options were discussed at the time of each specialists' consultation. The visit was facilitated by a clinic-specific advanced practice nurse and each patient received a patient-specific summary letter of treatment recommendations that included a list of recommended clinical trials (Figure 1). The clinic's advanced practice nurse reviewed the summary letter with each patient at the completion of the visit. Patients indicating interest in a clinical trial received either a same-day consultation with the research team or additional trialspecific print information.

To determine whether the clinical trial initiative increased clinical trial enrollment, the authors evaluated 1370 men with nonmetastatic localized prostate cancer seen at the MPCC from 2004 to 2008 and subsequently treated at MD Anderson Cancer Center. This date range was selected because it included men 2 years before and 2 years after the start of the trial initiative. The number of patients seen in the MPCC is larger after the start of the trial initiative because more appointment slots were available to patients in the later years. Each patient evaluated in the MPCC was self- or physician-referred and recently diagnosed (within 6 months) with localized disease, and had not yet received definitive treatment. The primary outcome was enrollment in a prostate cancer clinical trial, which were divided into 4 categories: (1) laboratory studies, (2) quality of life (QOL) studies, (3) procedure studies, and (4) novel studies (Table 1). Two physicians (K.E.H., D.A.K.) and a clinical nurse specialist (L.T.M.) categorized the trials. All 3 agreed on the final characterization for all trials.
The clinical trials that each patient enrolled in were obtained from the electronic medical record.

The covariates evaluated for association with enrollment in a prostate cancer clinical trial included age, selfreported race, residence, prostate cancer risk group, and year of visit. Age was evaluated as a continuous variable and was also dichotomized at the median (64.2 years) for descriptive statistics. Visit year was evaluated as both a continuous and a categorical variable. Patients with prostate cancer were categorized as either having visited the MPCC before or after the clinical trial enrollment initiative began in 2006. Residence was categorized as either within the Houston 10-county metropolitan area or outside of the metropolitan area. The Houston-Sugar Land-Baytown Metropolitan Statistical Area consists of Austin, Brazoria, Chambers, Fort Bend, Galveston, Harris, Liberty, Montgomery, San Jacinto, and Waller counties. Prostate cancer risk group was categorized by $\mathrm{NCCN}$ criteria as low-risk (stage T1a-T2a, Gleason score $\leq 6$, and prostate-specific antigen $[\mathrm{PSA}]<10 \mathrm{ng} / \mathrm{mL}$ ); high-risk (stage T3-4, Gleason score $\geq 8$, or PSA $>20$ $\mathrm{ng} / \mathrm{mL}$ ); or intermediate-risk (all others). ${ }^{8}$

Descriptive statistics were generated to characterize the study cohort. Chi-square statistic was used to compare categorical variables. Univariate and multivariate logistic regression evaluated the association between covariates and enrollment in a clinical trial. Results are reported as odds ratios $(\mathrm{OR})$ and adjusted $\mathrm{OR}(\mathrm{AOR})$ with $95 \%$ CIs (Table 2). $P$ values less than .05 were considered statistically significant. Analyses were conducted using SAS 9.2 (Cary, North Carolina).

\section{Results}

\section{Patient Characteristics}

A total of 1370 men with localized prostate cancer were seen at the MPCC between 2004 and 2008 and subsequently received treatment at MD Anderson. The median age at the time of visit was 64 years (interquartile range, 57.6-69.5). Most men were white (83\%) and lived outside the Houston metropolitan area (70\%; Table 3$)$. Thirty-two percent $(n=442)$ of men had low-risk disease, 49\% $(n=674)$ had intermediate-risk disease, and 19\% ( $\mathrm{n}=254)$ had high-risk disease. Of the 1370 patients, 326 (24\%) were seen in MPCC before the 2006 enrollment initiative began, and 1044 (76\%) were seen after the enrollment initiative was implemented. 
Clinical Trial Initiative

Dear Mr.

Thank you for choosing The University of Texas MD Anderson Cancer Center Multidisciplinary Prostate Cancer Clinic for your treatment consultation and recommendations. Dr. saw you in the clinic today, , Urologist, and Dr ,2013. These doctors and their staff are referred to as your team. Radiation Oncologist

The team has reviewed your medical history, biopsy, X-ray and lab tests, and the results of your physical exam. The doctors recommend the following treatment options, in no particular order, for your prostate cancer:

Surgery

$\square$ Open, retropubic radical prostatectomy (removal of the prostate)

$\square$ Robotic prostatectomy (less invasive procedure to remove the prostate)

$\square \quad$ With unilateral/bilateral nerve-sparing

口 Recommended clinical trials

Radiation Therapy

$\square \quad$ External-beam radiation therapy

$\square \quad$ Proton therapy

$\square \quad$ Brachytherapy (seed implant)

$\square$ With hormone therapy of

口 Recommended clinical trials

Active Surveillance (also called watchful waiting)

$\square \quad$ Recommended clinical trials

Cryotherapy (freezing of the prostate gland)

$\square \quad$ Recommended clinical trials

Hormone Therapy Alone

\section{About Clinical Trials}

Clinical trials are research studies that involve people. The main purpose of a clinical trial is to find a better way to prevent, diagnose, or treat a disease. Clinical trials are part of a long, careful research process. Patients who participate in a clinical trial receive drugs, procedures, or therapies that have already been researched in successful laboratory, animal, and/or human studies. All patients who participate in clinical trials are volunteers. They can choose to stop their participation in a clinical trial at any time.

Clinical trials are important to develop new treatments for cancer. Many of today's standard cancer treatments are based on the results of previous clinical trials. MD Anderson Cancer Center is committed to improving the treatment outcome of every cancer patient. If there is a clinical trial that would be applicable to your treatment recommendation, it has been listed after the treatment (above).

Figure 1 Summary letter provided to patients at the completion of the Multidisciplinary Prostate Cancer Clinic visit.

\section{Clinical Trial Enrollment}

Of the total study population, $73.5 \%(\mathrm{n}=1007)$ enrolled in at least one clinical trial and $28.5 \%(n=390)$ enrolled in 2 or more clinical trials; $66.9 \%$ of men enrolled in laboratory studies, $17.9 \%$ enrolled in QOL studies, $12.9 \%$ enrolled in novel treatment studies, and 3.7\% enrolled in procedural studies.

In multivariate analysis that adjusted for age, race, residence, and prostate cancer risk group, men seen in clinic after implementation of the clinical trials enrollment initiative were more likely to enroll in a clinical trial than those seen before implementation of the initiative (AOR, 8.22; 95\% CI, 6.16-10.96; $P<.001$ ). Over- all, the proportion of patients enrolling in clinical trials increased from 38.9\% (127/326) before the enrollment initiative to $84.3 \%(880 / 1044)$ after the initiative. Similarly, the proportion of men enrolling in 2 or more trials increased from $10.4 \%$ before the initiative to $34.1 \%$ after the initiative. Patient enrollment increased from $25 \%$ to $80 \%$ in laboratory studies, from $10 \%$ to $26 \%$ in QOL studies, and from 6\% to $15 \%$ in novel studies, and decreased from $8 \%$ to $2 \%$ in procedural studies (Figure 2). Similarly, when year was evaluated as a continuous variable, men seen in the MPCC clinic in later years were more likely to enroll in clinical trials $(\mathrm{AOR}, 2.33$; 95\% CI, 2.07-2.62; $P<.001$ per year increase). 
Madsen et al

\begin{tabular}{|ll|}
\hline Table 1 Clinical Trial Categories \\
\hline Category & Trials \\
\hline Laboratory & $\begin{array}{l}\text { Blood draws and tissue banking } \\
\text { trials }\end{array}$ \\
Quality of life & Questionnaire and survey studies \\
Procedure & $\begin{array}{l}\text { Trials evaluating new radiation and } \\
\text { surgical techniques } \\
\text { Novel }\end{array}$ \\
& $\begin{array}{l}\text { Trials evaluating investigational } \\
\text { treatments and systemic agents }\end{array}$ \\
\hline
\end{tabular}

Patient age at the time of MPCC was also found to be associated with clinical trial enrollment. Older patients were less likely to enroll in clinical trials than their younger counterparts (AOR, 0.97; 95\% CI, 0.95-0.98; $P<.001$ per year increase); 79\% of men younger than 64.2 years were enrolled in a clinical trial compared with only $68 \%$ of men older than 64.2 years.

Multivariate analysis also identified a trend for patients in the highest prostate cancer risk group to be more likely to participate in clinical trials than low-risk patients (AOR, 1.43; 95\% CI, 0.96-2.13; $P=.083)$. However, unadjusted absolute differences were small, with $74.4 \%$ of high-risk patients and $73.5 \%$ of low-risk patients participating in clinical trials.

\section{Discussion}

MD Anderson noted a significant increase in clinical trial enrollment, related to referrals from the MPCC, after the introduction of the clinical trial initiative. The authors believe that the success of this initiative can be attributed to the structure of the multidisciplinary clinic, which incorporated a collaborative approach to recommending clinical trials during each specialist's consultation. The clinic is designed for long counseling visits, during which all treatment options are discussed while providing detailed patient education on the appropriateness of a recommended trial. The initiative also included posting information about specific protocols and eligibility criteria in the clinic area for physician reference, the addition of an advanced practice nurse to facilitate clinic visits, a patient-specific summary letter of treatment recommendations that included a list of recommended clinical trials (Figure 1), trial-specific print information, and same-day consultation with clinical trial research teams.

Notably, after the trial initiative began, clinical trial enrollment in the MPCC increased across multiple trial types, including those that evaluated investigational treatments, systemic agents, and QOL. The largest increase, however, was observed in the trials that collected and banked blood and tissue for future study. Because time constraints and travel inconveniences

\section{Table 2 Factors Associated With Clinical Trial Enrollment in Univariate and Multivariate Analysis}

\begin{tabular}{|c|c|c|c|c|c|}
\hline \multirow[b]{2}{*}{ Characteristic } & \multirow{2}{*}{$\begin{array}{l}\text { Percent Enrolled } \\
\text { in a Clinical Trial }\end{array}$} & \multicolumn{2}{|c|}{ Univariate Analysis } & \multicolumn{2}{|c|}{ Multivariate Analysis } \\
\hline & & OR $(95 \% \mathrm{Cl})$ & $P$ Value & AOR $(95 \% \mathrm{Cl})$ & $P$ Value \\
\hline \multicolumn{6}{|l|}{ Age } \\
\hline Per year increase & - & $0.97(0.95-0.98)$ & $<.0001$ & $0.97(0.95,0.98)$ & $<.0001$ \\
\hline \multicolumn{6}{|l|}{ Race/ethnicity } \\
\hline Caucasian & $73.9 \%$ & Reference group & & Reference group & \\
\hline African American & $76.0 \%$ & $1.12(0.75-1.68)$ & .572 & $0.94(0.59,1.48)$ & .779 \\
\hline Other & $65.2 \%$ & $0.66(0.42-1.04)$ & .074 & $0.56(0.34,0.94)$ & .027 \\
\hline \multicolumn{6}{|l|}{ Prostate cancer risk group } \\
\hline Low risk & $73.5 \%$ & Reference group & & Reference group & \\
\hline Intermediate risk & $73.1 \%$ & $0.98(0.75-1.29)$ & .887 & $1.16(0.85,1.58)$ & .346 \\
\hline High risk & $74.4 \%$ & $1.05(0.74-1.49)$ & .799 & $1.43(0.96,2.13)$ & .083 \\
\hline \multicolumn{6}{|l|}{ Residence } \\
\hline Within Houston metropolitan area & $81.4 \%$ & Reference group & & Reference group & \\
\hline Outside Houston metropolitan area & $70.0 \%$ & $0.54(0.40-0.71)$ & $<.0001$ & $0.80(0.58,1.10)$ & .176 \\
\hline \multicolumn{6}{|l|}{ Clinic visit } \\
\hline Before clinical trial initiative & $38.9 \%$ & Reference group & & Reference group & \\
\hline After clinical trial initiative & $84.3 \%$ & $8.41(6.37-11.10)$ & $<.001$ & $8.22(6.16-11.0)$ & $<.001$ \\
\hline
\end{tabular}

Abbreviations: AOR, adjusted odds ratio; OR, odds ratio. 
Clinical Trial Initiative

\begin{tabular}{|c|c|}
\hline Characteristic & Distribution \\
\hline \multicolumn{2}{|l|}{ Age, $y$} \\
\hline Median (range) & $64.2(41.0-86.8)$ \\
\hline \multicolumn{2}{|l|}{ Race/ethnicity, $n$} \\
\hline White & $1132(82.6 \%)$ \\
\hline African American & $146(10.7 \%)$ \\
\hline Other & $92(6.7 \%)$ \\
\hline \multicolumn{2}{|l|}{ Prostate cancer risk group, $n$} \\
\hline Low risk & $442(323.0 \%)$ \\
\hline Intermediate risk & $674(49.2 \%)$ \\
\hline High risk & $254(18.5 \%)$ \\
\hline \multicolumn{2}{|l|}{ Residence, $n$} \\
\hline Within Houston metropolitan area & $419(30.6 \%)$ \\
\hline Outside Houston metropolitan area & $951(69.4 \%)$ \\
\hline \multicolumn{2}{|l|}{ Clinic visit, $n$} \\
\hline Before clinical trial initiative & $326(23.8 \%)$ \\
\hline After clinical trial initiative & $1044(76.2 \%)$ \\
\hline
\end{tabular}

can be an obstacle to trial enrollment, ${ }^{9}$ the authors speculate that this latter enrollment increase was partly because of efforts to minimize additional patient effort, such as the collection of banked blood in combination with PSA assessments and the retrieval of prostate tissue during the scheduled prostatectomy, if patients were agreeable.

Lack of physician and patient awareness of available trials has been identified as a barrier to clinical trial enrollment. ${ }^{5}$ Increasing physician and patient awareness of available clinical trials through postings in the clinic and inclusion of a list of recommended clinical trials (Figure 1) in the summary letter provided to patients with prostate cancer at the completion of the multidisciplinary clinic visit are key reasons why the present intervention was successful.

Providing clinical trial counseling and recommendations in a multidisciplinary setting that allowed for long counseling visits and collaborative decision-making also contributed to the success of the clinical trial intervention. Physician recommendation of clinical trials is known to increase trial enrollment, ${ }^{10,11}$ and most patients prefer a collaborative approach to clinical trial decision-making. ${ }^{12}$ Wallace et $\mathrm{al}^{13}$ successfully increased accrual to a difficult-to-recruit-for clinical trial after adding a multidisciplinary education session presented by a urologist and radiation oncologist as part of the recruitment process.
Factors beyond patient education are also instrumental in increasing study enrollment and participation. Jacobs et $\mathrm{al}^{5}$ found 3 factors to be associated with increased cancer trial enrollment: dedicated research staff, recognition of physicians who consistently enrolled patients, and the number of clinical trials available for consideration. The present successful trial initiative did have dedicated staff, including the physician, and the clinic advanced practice nurse, and multiple trials were available for participation. Davison et $\mathrm{al}^{11}$ describe factors identified by patients as influential in their decision to participate in prostate cancer protocols. These factors varied from an altruistic intent to help future patients diagnosed with prostate cancer, to the potential impact of study treatment on survival and/or quality of life, and the relationship and/or personal recommendation by the urologist, medical oncologist, or radiation oncologist. Receiving "full and open information" to provide informed consent was also a primary influence on patients' decisions to participate. The present successful clinical trial initiative offered detailed information about eligible clinical trials and provided specific written recommendations from the clinicians. Patients may attribute reservations toward clinical trial participation as concerns about not receiving standard of care treatment. ${ }^{9}$ However, after the introduction of the clinical trial initiative, a 2.5-fold increase in enrollment in investigational treatments and systemic agents was observed at this center.

Although prostate cancer is more common among older men, the authors found that older men were less likely to enroll in clinical trials. The discrepancy between the age of patients enrolling in cancer research trials and the age of patients in the general population with a cancer diagnosis has been documented in other studies. ${ }^{7,14}$ Prostate cancer is not only the second leading cause of cancer death among men but also a disease of aging. With nearly $60 \%$ of new cancers and more than $70 \%$ of cancer deaths occurring in the population aged 65 years and older, ${ }^{2,15}$ advances in prostate cancer treatment depend on a representative sample of an older population in clinical trials. The authors believe future prostate cancer trials should make an effort to enroll patients aged 65 years and older.

The authors acknowledge several study limitations. Availability of protocols is known to impact protocol enrollment, ${ }^{2}$ and the available protocols changed over the study period because protocols were continually opening and closing. It must also 


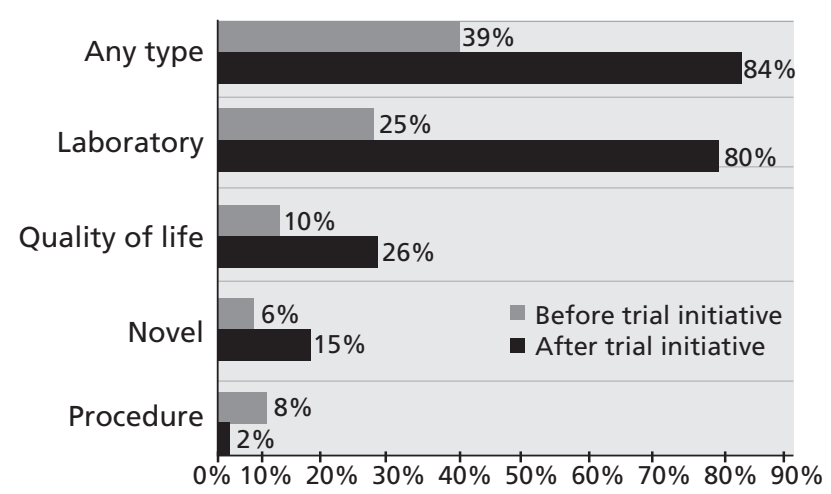

Figure 2 Patient enrollment in clinical trials before and after the clinical trial initiative (see Table 1 for clinical trial category types).

be noted that although the authors were able to identify the number of protocols that were accepted by patients per year throughout the initiative, it was not possible to document all trials offered to patients throughout this initiative. Therefore, the number of trials offered to patients before and after the initiative could not be quantified, but the number of trials patients enrolled in was similar before and after the initiative (17 various trials before the initiative and 19 various trials after), suggesting that the number of available trials was relatively consistent across the study period. The authors also note that although grant funding and financial support may contribute to increased laboratory investigations using patient specimens and have a subsequent impact on trial enrollment, they were not aware of any significant financial grants or administrative support changes to their institutional infrastructure before or after the trial initiative. Another limitation was that although the frequency of clinical trial enrollment was assessed, patients were not asked why they enrolled in clinical studies, and therefore the authors acknowledge the inability to assess how appealing specific trials were to individual patients or whether press coverage of interventions evaluated in the trials may have influenced trial enrollment. Finally, the authors note that the decrease in enrollment in procedural studies after the introduction of the clinical trial initiative was related to fewer procedural studies available for enrollment during that period.

\section{Conclusions}

The authors believe that the increase in clinical trial enrollment to $84 \%$ in at least one clinical trial and to $34 \%$ in 2 or more clinical trials after the start of the clinical trial initiative highlights the effect that focused efforts toward trial enrollment may have on the current national averages of less than $5 \%$ of patients with cancer. The results of this clinical trial initiative support the recommendation to develop a comprehensive strategy to ensure that physicians are knowledgeable of all available protocols, to educate patients about appropriate diseasespecific clinical trials during the initial consultation, and to streamline the process for clinical trial referrals to accommodate patient schedules.

\section{References}

1. What are the key statistics about prostate cancer? American Cancer Society Web site. Available at: http://www.cancer.org/cancer/ prostatecancer/detailedguide/prostate-cancer-key-statistics. Accessed June 4, 2014.

2. Siegel R, Naishadham D, Jemal A. Cancer statistics, 2013. CA Cancer J Clin 2013;63:11-30.

3. Resnick MJ, Koyama T, Fan KH, et al. Long-term functional outcomes after treatment for localized prostate cancer. N Engl J Med 2013;368:436-445.

4. Gansler T, Jin M, Bauer J, et al. Outcomes of a cancer clinical trial matching service. J Cancer Educ 2012;27:11-20.

5. Jacobs SR, Weiner BJ, Minasian LM, et al. Achieving high cancer control trial enrollment in the community setting: an analysis of the Community Clinical Oncology Program. Contemp Clin Trials 2013;34:320-325.

6. Janet Yang Z, McComas K, Gay G, et al. From information processing to behavioral intentions: exploring cancer patients' motivations for clinical trial enrollment. Patient Educ Couns 2010;79:231-238.

7. Comis RL, Aldige CR, Stovall EL, et al. A quantitative survey of public attitudes towards cancer clinical trials. Available at: http:// www.cancertrialshelp.org/CTHpdf/308-9.pdf. Accessed June 17, 2014.

8. Mohler JL, Kantoff PW, Armstrong AJ, et al. NCCN Clinical Practice Guidelines in Oncology: Prostate Cancer. Version 2.2014. Available at: NCCN.org. Accessed June 26, 2013.

9. Patlak M, Nass S. Improving the Qualiy of Cancer Clinical Trials: Workshop Summary National Cancer Policy Forum. Washington DC: The National Academies Press; 2008.

10. Heiney SP, Arp Adams S, Drake BF, et al. Successful subject recruitment for a prostate cancer behavioral intervention trial. Clin Trials 2010;7:411-417.

11. Davison BJ, So A, Goldenberg SL, et al. Measurement of factors influencing the participation of patients with prostate cancer in clinical trials: a Canadian perspective. BJU Int 2008;101:982-987.

12. Biedrzycki BA. Factors and outcomes of decision making for cancer clinical trial participation. Oncol Nurs Forum 2011;38:542-552.

13. Wallace $K$, Fleshner $\mathrm{N}$, Jewett $\mathrm{M}$, et al. Impact of a multi-disciplinary patient education session on accrual to a difficult clinical trial: the Toronto experience with the surgical prostatectomy versus interstitial radiation intervention trial. J Clin Oncol 2006;24:4158-4162.

14. Gross CP, Herrin J, Wong N, et al. Enrolling older persons in cancer trials: the effect of sociodemographic, protocol, and recruitment center characteristics. J Clin Oncol 2005;23:4755-4763.

15. Smith AW, Reeve BB, Bellizzi KM, et al. Cancer, comorbidities, and health-related quality of life of older adults. Health Care Financ Rev 2008;29:41-56. 